\title{
Quelques remarques et une enquête sur un modèle pour l'écriture en milieu scolaire
}

\section{Rosine Galluzzo-Dafflon}

\section{OpenEdition}

\section{Journals}

Édition électronique

URL : https://journals.openedition.org/trema/3491

DOI : 10.4000/trema.3491

ISSN : 2107-0997

\section{Éditeur}

Faculté d'Éducation de l'université de Montpellier

\section{Édition imprimée}

Date de publication : 1 octobre 2016

Pagination : 19-30

ISBN : 979-10-96627-01-1

ISSN : 1167-315X

\section{Référence électronique}

Rosine Galluzzo-Dafflon, «Quelques remarques et une enquête sur un modèle pour l'écriture en milieu scolaire », Tréma [En ligne], 45 | 2016, mis en ligne le 01 janvier 2017, consulté le 07 décembre 2022. URL : http://journals.openedition.org/trema/3491; DOI : https://doi.org/10.4000/trema.3491

Ce document a été généré automatiquement le 29 septembre 2020.

Tous droits réservés 


\title{
Quelques remarques et une enquête sur un modèle pour l'écriture en milieu scolaire
}

\author{
Rosine Galluzzo-Dafflon
}

1 Je me propose de soumettre à la discussion un modèle schématique pour l'écriture en milieu scolaire (annexe 1) extrêmement répandu dans les milieux de la formation. J'évoquerai en premier lieu son contexte d'émergence et quelques-unes des critiques dont il a pu faire l'objet, de manière plus ou moins explicite. Dans un deuxième temps, mon questionnement se centrera sur un aspect moins controversé de ce modèle: la représentation du temps (instant, durée, rythme). Ainsi, à partir de deux séries d'observation opérées en CM2 dans le but d'analyser la manière dont le temps est utilisé pour mener certaines activités de français, je soulignerai l'importance des principes permettant d'organiser le temps didactique dédié aux pratiques d'écriture scolaires. Ce commentaire me conduira à préciser à quelles conditions de telles pratiques pourraient effectivement constituer un processus générant des apprentissages, autrement dit à pointer les limites du modèle.

\section{Un modèle pour l'écriture en milieu scolaire}

\section{1. Contexte d'émergence}

2 Le schéma dont il est ici question a été proposé en 1990 par Claudine Garcia-Debanc dans son ouvrage L'élève et la production d'écrits. Il a été repris tel quel en 1992 par Jean-François Halté dans un numéro de la collection Que sais-je ? consacré à la didactique du français. 
Mais il a été largement préparé par une publication de Maurice Mas, en 1987, dans un numéro de la revue Repères intitulé Des critères pour écrire.

Ce modèle a donc été élaboré au sein d'une recherche-action INRP Français (1983) portant sur la lecture et l'écriture et destinée à :

- mettre en place, dans les classes élémentaires, des pratiques d'évaluation formative des écrits fondées sur des théories linguistiques et psycholinguistiques

- en définir les traits spécifiques par rapport aux pratiques d'évaluation traditionnelles

- en évaluer les effets sur les apprentissages des élèves

Or, chez Claudine Garcia-Debanc, ce modèle est appelé Schéma systémique de la production écrite et s'organise autour d'un axe central qui va de l'écrire au réécrire. Chez Halté, il accompagne un paragraphe intitulé Un modèle de l'activité d'écriture, dans la partie conclusive du dernier chapitre du Que sais-je? rappelant deux principes majeurs: l'apprentissage des savoirs et savoir-faire s'effectue dans et par l'action ; c'est l'élève, acteur dans toutes les cases du schéma, qui apprend et qui situe par là-même les tâches de l'enseignant. On peut donc relever un glissement du domaine d'application du modèle d'origine qui, parti pour expliquer des procédures d'évaluation formative organisées autour de l'élaboration de critères (textuels ou de réalisation), en vient à décrire ${ }^{1}$ un processus, voire le processus de l'écriture en milieu scolaire et, finalement, l'organisation de la classe de Français, associant les composantes de sa configuration disciplinaire (écriture, lecture, oral, étude de la langue).

\section{2. Mises en question}

5 Peut-être peut-on voir dans ce glissement de fonction l'origine de certains questionnements du Schéma systémique de la production écrite ou de son opérationnalisation dans les classes. Ces questionnements sont nombreux. Je n'en retiendrai que trois ensembles venus d'auteurs qui, à une période très fortement préoccupée par l'enseignement et l'apprentissage de l'écriture à l'École, portent sur elle des regards différents mais, on le verra, complémentaires. C'est ainsi que Claudine Garcia-Debanc (1990) attire l'attention sur certains risques liés à l'élaboration des critères. Ces risques consistent en l'atomisation de la compétence scripturale sous l'effet d'une multitude de critères définis pour réaliser la tâche, en une répartition inefficace du temps didactique entre constitution des critères et écriture proprement dite ou encore en des normalisations excessives bridant l'imagination. Sont également pointés le défaut d'autonomie auquel on expose les élèves en mettant à leur disposition trop tôt trop d'outils pour accompagner l'écriture ainsi que la nécessité de constituer d'emblée l'écriture en situation-problème, qui fasse sens et en justifie le processus.

6 Yves Reuter (2002-2003) interroge plus généralement les modélisations de l'écriture en didactique du français - c'est-à-dire de « l'écriture en tant qu'elle s'enseigne ou s'apprend » (p. 22 ) - et les conceptions sur lesquelles elles reposent, notamment l'image de référence de l'écriture, autrement dit « la conception globale, souvent implicitée, de son fonctionnement » (p. 26) qu'on en a. Même s'il ne s'intéresse pas directement au modèle spécifique des processus d'écriture proposé par Mas et Garcia-Debanc, il pose, entre autres questions ${ }^{2}$, celle de savoir «jusqu'à quel point un modèle de l'écriture, en tant qu'il demeure isolé, présente une réelle pertinence didactique» (p. 21). Ainsi, en opposant le faire comme activité et comme pratiques, il attire l'attention sur deux conceptions antagonistes de l'écriture qui commandent tantôt de la considérer à travers des invariants, comme une activité 
décontextualisée et donc possiblement unique (ce qu'invite à faire le schéma de Mas), tantôt, au contraire, comme une pratique socialement différenciée, liée aux représentations et aux pratiques sociales et donc nécessairement plurielle et diverse. Pour Reuter, ce sont des modes d'articulation de ces deux conceptions qui sont à rechercher.

Quant à Jean-Charles Chabanne et Dominique Bucheton (2002), ils s'intéressent prioritairement au sujet écrivant dans une perspective culturelle. Pour eux, la notion de modèle didactique recouvre, de façon large, les «manières d'organiser les activités d'écriture en classe, de proposer des tâches d'entrainement et d'évaluation, de lire et d'évaluer les copies, d'organiser corrections et remédiations" (p. 25), c'est-à-dire un ensemble de choix théoriques, de démarches et de valeurs le plus souvent tacites. Dans la classe, une priorité est donc à donner aux expériences d'écriture-lecture, aux rencontres avec des écrits motivants et modélisants. Mais, contrairement à ce qu'induit la mise en œuvre du Schéma systémique de la production écrite, il s'agit moins de se préoccuper du produit fini de l'écriture que de son dynamisme. L'activité est comprise au sens psychologique, comme un ensemble complexe d'actions simultanées et intriquées, qui ne se réduisent pas à la dimension linguistique des productions mais travaillent le rapport singulier de l'élève à l'écrit. En ce sens, la réécriture se distingue de la révision ou de la correction du premier jet, pour devenir moyen d'élaboration (parfois douloureuse, disent-ils) d'un écrit et du sens. On le voit, ces différents points de vue sur l'écriture scolaire opèrent comme autant de coups de projecteur qui en éclairent de multiples composantes, opérations, rapports à..., en mettant l'accent soit sur la dimension linguistique des productions (discours, genre), soit sur leur dimension sociologique ou psychologique, déterminée par le sujet scripteur.

\section{La question du temps et de la durée dans le modèle}

\section{1. Une durée linéaire?}

Pour ce qui me concerne, je m'intéresserai prioritairement à la manière dont le modèle rend compte du temps dans lequel s'inscrit l'écriture scolaire. En choisissant une telle entrée, je garde toutefois pour projet qu'elle me reconduise aux questionnements que je viens de rappeler. Ainsi, vis-à-vis du temps de l'écriture, je poserai d'emblée trois remarques (annexe 1):

- le temps semble s'absenter des verbes qui constituent l'axe horizontal du schéma, verbes d'action (écrire, lire, relire, repérer) utilisés à l'infinitif pour caractériser la dynamique de l'écriture en général, comme activité et non comme pratiques ;

- cependant, la durée du processus d'écriture apparaît comme une durée linéaire, une suite d'opérations réunies par des liens de causalité : c'est parce qu'on a écrit des textes que l'on peut les relire dans la classe, c'est parce qu'on les relit qu'on peut repérer des dysfonctionnements, etc.;

- cette linéarité se produit à son tour par le concours d'une double circulation périphérique, verticale celle-là : d'abord autour du premier jet (écrire), inscrit dans un projet qu'accompagnent la lecture, les critères et divers outils; ensuite, autour de la réécriture, alimentée par des activités décrochées.

9 Je propose ici de lire ces deux axes à travers la distinction, chez Bachelard, de l'instant et de la durée, puis du temps vécu et du temps pensé, autrement dit de problématiser (Fabre et Musquer, 2009) le temps didactique. Ainsi s'agira-t-il d'abord, en marge de toute 
considération d'ordre épistémologique concernant l'objet de l'écriture (interview, récit), de définir à quelle(s) condition(s) les différentes données (textes, outils, activités) qui apparaissent dans la succession des séances consacrées à l'écriture en classe peuvent effectivement, sur l'ensemble d'une séquence ou période, permettre aux élèves de produire un écrit pertinent ${ }^{3}$. C'est bien l'écriture scolaire, dans toute sa spécificité, qui se trouve ici problématisée. J'entendrai par temps didactique l'ensemble des moments d'une séquence d'enseignement et d'apprentissage au cours desquels le professeur organise des activités spécifiques en vue de faire construire aux élèves certains savoirs et compétences en écriture. Quelles sont les caractéristiques de ce temps didactique?

\section{2. Instant et durée chez Bachelard}

10 Contre la thèse bergsonienne du temps, Bachelard $(1931,1950)$ considère que « la vraie réalité du temps, l'unité, c'est l'instant » (1931, p. 25). La durée n'est qu'une construction a posteriori, une imagination voire une illusion. Sa trame ne se construit qu'au rythme de la nouveauté qui apparait dans la succession des instants. "Elle est une poussière d'instants, mieux, un groupe de points qu'un phénomène de perspective solidarise plus ou moins étroitement " (p. 33). La ligne qui réunit les points et qui schématise la durée n'est donc qu' "une fonction panoramique et rétrospective » (p. 33), au caractère subjectif indirect et secondaire. Bachelard oppose également temps vécu et temps pensé. Le temps vécu, ou temps du monde, a une dimension horizontale : c'est le temps immanent. Le temps pensé, lui, est le temps du moi, avec une dimension verticale : c'est le temps transitif. Et ces deux temps ne sont pas naturellement synchrones. Or, le temps vécu est un temps inefficace, parce que dispersé en une poussière d'instants hétéroclites. Seul le temps pensé, subjectif, peut-être un temps cohéré, organisé, consolidé en durée. Le temps didactique apparaît alors comme hautement problématique, dans le sens où l'élève qui apprend à écrire doit trouver dans les séances discontinues qui constituent le décousu temporel et spatial de la classe de quoi reconstruire une logique, qui ne peut que lui être propre. Une telle problématisation nécessite donc que l'écriture scolaire, quelle que soit la production visée, s'inscrive dans un temps didactique qui obéit à certaines conditions.

11 Pour déterminer quelques-unes de ces conditions, je m'appuierai sur deux séries d'observation réalisées dans un CM2 en centre-ville et un CM2 en milieu rural. Sans viser l'exhaustivité, compte tenu du caractère aléatoire et limité des exemples de pratiques retenus, je m'attacherai à dégager quelques principes généraux susceptibles de concerner toute pratique de l'écriture bornée par les impératifs du temps didactique. Les séances d'écriture observées ont été menées par des professeurs non débutants, auprès de groupes d'élèves comparables en nombre. Les deux professeurs (un homme, une femme) ont été préalablement rencontrés pour un premier échange où ils ont eu l'occasion d'expliciter ce que leurs pratiques d'enseignement de l'écriture doit au modèle de Mas. Ils ont également été rencontrés après les séances d'observation, pour un entretien d'explicitation portant sur les activités qu'ils avaient conduites pendant la séquence. Ces activités s'inscrivent dans des périodes respectivement ponctuées par l'écriture d'une interview et d'un récit d'aventures, et dans le cadre plus général d'un travail sur le journal ou à partir du roman L'œil du Loup (Pennac). La séquence en centre-ville a ainsi donné lieu, entre le 27 novembre et le 18 décembre, à quatre observations portant sur les moments didactiques plus spécialement dédiés à l'écriture de l'interview (écriture du questionnaire, réalisation de la carte mentale, écriture puis réécriture de l'interview); sur le 
même principe, l'observation de la séquence en milieu rural a privilégié la rédaction des premiers jets du récit d'aventure (12 décembre) puis sa réécriture (17 décembre). À partir de cahiers ou classeurs d'élèves et de documents fournis par les professeurs, la description intégrale des deux séquences a fait l'objet d'une mise au point concertée, sous la forme d'un tableau récapitulant la date et la durée de chaque séance, le contenu des activités ainsi que les outils, les supports utilisés et les traces obtenues. Des diagrammes analysant le pourcentage du temps didactique accordé à chaque type d'activités dans les séances observées ${ }^{4}$ ont été construits.

Dans les deux cas, le temps vécu apparaît effectivement comme une poussière d'instants discontinus du seul fait de la durée de la séquence et/ou de son découpage en séances organisées entre elles : dans le CM2 en centre-ville, la séquence « interview » s'étend du 25 septembre au 19 décembre 2014, soit une période de trois mois et treize séances de quarante-cinq à cinquante-cinq minutes chacune; dans le CM2 en milieu rural, la séquence «L'œeil du loup » ne dure qu'un mois, du 20 novembre au 20 décembre 2014, mais elle occupe onze séances de quarante-cinq à quatre-vingt-dix minutes chacune. L'écriture s'y produit plutôt en fin de séquence, comme application/imitation de ce qui a été travaillé auparavant. Ces deux exemples de mises en œuvre de l'écriture scolaire, qui sont assez représentatifs des pratiques «ordinaires " d'écriture au cycle 3, me serviront de supports pour comprendre comment on peut faire cohérer l'ensemble des activités ( écriture, lecture, oral) autour d'une production écrite, les organiser en un tout signifiant pour l'élève. Autrement dit, quel sens peuvent prendre au fil des semaines les séances dédiées à l'écriture ? Et comment, d'une séance à l'autre (parfois éloignée dans le temps), chaque élève peut-il progresser dans la construction de la réponse apportée au problème posé par l'écriture ${ }^{5}$ ?

\section{Deux exemples de scénarisation du temps de l'écriture en $\mathrm{CM} 2$}

\section{1. Problématiser l'écriture pour assurer la liaison des instants}

13 L'analyse des tableaux récapitulatifs et des diagrammes élaborés en concertation à l'issue de la période d'observation permet de dégager deux scénarios de séquence, c'est-à-dire deux canevas (ou trames) correspondant au compromis obtenu entre le projet didactique de l'enseignant et la manière dont les élèves de CM2 se le sont approprié, dans le déroulement effectif des activités en classe. Ainsi la séquence en centre-ville se donne-telle pour finalité d'écrire une série d'interviews pratiquées sur différents membres de la communauté scolaire (personnel de restauration, animatrice, professeur de musique, stagiaire, titulaire, responsable des Temps d'Activités Péri-éducatives'). Ces interviews visent à mieux faire connaître les individus choisis. Au moment de l'écriture, ce sont en premier lieu les échanges progrédiants (tournés vers l'avenir du projet) et récurrents (prenant en compte ce qui a déjà été fait) qui, dès l'ouverture de la séance, assurent le tissage (Bucheton, 2014) entre les activités, au rythme moyen d'une séance par semaine. Ces échanges fonctionnent comme des rituels, des routines, opérant non seulement un lien entre un avant et un après (la lecture de la Une et de divers articles de journaux et l'écriture d'une interview, par exemple) mais entre le dedans (la classe) et le dehors (l'École). Ainsi l'écriture n'y est-elle plus activée comme un exercice scolaire individuel destiné à la seule lecture évaluative du professeur, mais comme une pratique sociale, une communication 
réellement instanciée entre les élèves et l'École, qui œuvre pour une mutuelle (re)connaissance des acteurs de la vie scolaire ${ }^{7}$.

Mais en ravivant régulièrement des traces de déjà-là, le tissage de début de séance ramène à l'instant inaugural d'un projet plus ambitieux, défini en accord avec les élèves : la mise en ligne régulière de leurs productions, sous la forme d'un journal scolaire. $\mathrm{Ce}$ moment important, qui marque la suprématie du temps voulu sur le temps vécu, correspond au surgissement d'un problème: qu'est-ce qui vaut la peine d'être écrit à l'école et, plus particulièrement ici, qui «mérite » d'être interviewé ${ }^{\text {? Pourquoi et pour }}$ qui le fait-on? De façon délibérée, pour fonder leur démarche d'enquête, le professeur demande aux élèves de partir du familier (les différents acteurs de l'école). Ce choix, au dire de Fabre (2014) citant Dewey, peut paraître judicieux puisque «le familier est gros de problèmes et [que] ce sont ces problèmes qui suscitent des enquêtes, lesquelles, convenablement orientées, débouchent sur des apprentissages » (p. 101). Or Fabre précise que «ce n'est pas dans le familier comme tel que s'origine la recherche » mais que le familier est «ce grâce à quoi l'élève peut porter son attention sur d'autres objets » (p. 101). Ainsi, dans l'entretien post-séquence, le professeur souligne-t-il que les interviews écrites sont finalement d'inégale qualité et que c'est la rencontre avec le tout nouveau responsable des TAP, peu connu des élèves, qui est à l'origine de la production la plus pertinente, cet acteur de la vie scolaire étant, à ce moment précis, véritablement objet de leur curiosité. Problématiser la séquence consacrée à l'écriture d'interviews, c'est donc se poser d'emblée des questions sur la finalité, les modalités et le contenu de ce qui est à écrire, c'est prendre garde à porter son attention sur une personne dont la présence suscite l'étonnement, fait question et dont les propos serviront de réponses à de « vraies » questions. Et ce suffisamment longtemps pour que le différé de l'écriture et du temps didactique ne le fragilise pas. Autrement dit, les enjeux de l'interview doivent être importants.

Une telle démarche peut, comme l'envisage le professeur, encourager à se saisir de toutes les données utiles de la vie scolaire et à instaurer des échanges entre les acteurs de l'école, de manière à nourrir dans l'année le projet plus vaste de la création d'un blog. Bucheton (2014) a précisément montré les bénéfices, en termes d'implication et de conceptualisation, de cette écriture collaborative au sein de la communauté discursive de la classe, écriture qui suppose une posture de lâcher-prise de l'enseignant et une chronologie moins formelle que celle d'une méthode. Mais, comme le conçoit le professeur dans l'entretien post-séquence, le blog pourrait devenir une interface permettant d'asseoir une complémentarité entre l'école et les familles. Cependant, pour devenir réellement un problème capable d'engager des apprentissages, la création de ce blog impose qu'on réfléchisse à la façon dont tous les acteurs (y compris les familles) peuvent se saisir de ces données', les commenter, y répondre..., et que l'on pense explicitement les utilisations, en classe, de ces retours. À terme, c'est bien la cohérence du temps et de la vie scolaires en CM2, la liaison des différents instants et lieux constitutifs du quotidien qui peuvent être données à lire non seulement au professeur et aux élèves mais à leurs parents, ce qui représente une autre façon de tisser le dedans et le dehors de l'institution scolaire.

\section{2. Construire et faire construire activement une durée}

16 Une telle cohérence est une condition permanente. Dans le CM2 en centre-ville, par exemple, l'éparpillement des instants didactiques est rendu sensible par la spécificité de 
la deuxième séance observée, structurée de manière originale : celle-ci est commandée par un obstacle organisationnel, tous les élèves n'ayant pas réussi à collecter les données nécessaires pour opérer la transcription de l'interview. Le professeur décide alors de leur faire dresser une carte mentale ${ }^{10}$ (annexe 2) dont il pointe dans l'entretien deux fonctions majeures : son rôle de tissage par rapport à des séances (les types d'articles, l'interview) qu'il juge « denses » sur le plan de l'information et de la trace écrite; son rôle d'outil pour la répartition du rôle des élèves dans la rencontre, à l'oral. Mais cette activité, qui s'apparente à un recueil de représentations, pourrait en outre, comme l'explicite a posteriori le professeur, permettre de préciser, à l'écrit, les finalités communicationnelles de l'interview. C'est ainsi qu'un travail collectif à partir de la catégorie "personne» inciterait, en introduction, à préciser l'identité de l'interviewé : pourquoi l'avoir choisi, lui ? pour obtenir quelles informations le rencontre-t-on? Quant à la catégorie «journal », en attirant l'attention sur les notions d' "information » et de "réponse », elle encourage les élèves à induire une appréciation conclusive qui vient donner son sens à la rencontre, tout en évitant une redondance avec les phrases introductives. Ainsi, l'irruption de l'imprévu, l'accident (Bachelard, 1950), loin de constituer une coupure dans le temps didactique, peut lui apporter un étayage précieux: l'outil dont il occasionne la construction s'avère productif non seulement à l'oral, pour réaliser la tâche, mais à l'écrit, en donnant des éléments de structuration de la production (introduction/conclusion ).

17 De même, dans la séquence "L'œil du loup ", les activités d'écriture et de réécriture, proches dans le temps, mobilisent fortement l'étayage du professeur qui alterne (Bucheton, 2014) une posture d'accompagnement et une posture de contrôle. L'analyse des premiers jets, comme l'évaluation magistrale et son compte-rendu en classe, fait cependant apparaître trois dysfonctionnements récurrents dans les récits d'aventure obtenus - mais assez peu fréquents d'autres années, selon le professeur, dans l'entretien post-séquence. Deux d'entre eux concernent l'action: ou bien celle-ci se réduit à un sommaire (une suite d'aventures fortement résumées) ou bien l'action, stéréotypée, est fortement redevable à l'œuvre littéraire étudiée (confrontation protagoniste/chasseurs; déplacement du protagoniste de l'Afrique à l'Europe). On peut penser que la phase d'évaluation magistrale pourrait alors utilement viser le recours à des outils permettant d'opérer auprès des élèves la dévolution des deux postures (accompagnement, contrôle) assumées ici par le professeur. Ainsi, de façon comparable à ce qui a pu être proposé en SVT (Orange, 2007; Chalak, 2014) ${ }^{11}$, on aurait recours à des caricatures. Dans le contexte de cette production d'écrit, il s'agirait de récits archétypiques présentant l'un des trois types de dysfonctionnements repérés dans les productions d'élèves mais récrits, stylisés, épurés par le professeur et, en ce sens, dépersonnalisés pour mieux faire saillir le problème rencontré. Ces caricatures, débattues par les élèves, donneraient l'occasion d'affiner un critère déjà formulé et d'inscrire la réécriture dans un enchaînement problématique, autrement dit une suite ordonnée de problèmes ${ }^{12}$, nécessairement liés entre eux. À titre d'exemple (non normatif), le critère "le texte est écrit en respectant les cinq étapes du récit ${ }^{13}$ " pourrait, au moment de la réécriture, devenir : «L'action raconte en détail une seule aventure survenue à X, dans/à... (un lieu précis). Dans cet épisode, X s'oppose à... et il est aidé par... ». 


\section{3. Construire une progression}

18 Le dernier principe capable de transformer le temps vécu en temps pensé revient à interroger les principes de progression actifs dans la classe. Selon Elisabeth Nonnon (2010), à une logique de distribution des contenus il s'agit de préférer une logique de développement de la pratique de l'écriture, articulant savoir et savoir-faire et nécessitant la sélection de notions-noyaux et de compétences prioritaires. Cette logique agit par approximations rectifiées au cours du temps, ce qui impose de définir des niveaux de formulation pour les différentes notions et des degrés d'exercices pour chaque compétence.

19 C'est ainsi que, pour ce qui concerne l'apprentissage du portrait, pratique requise au début des récits d'aventure, le professeur, dans l'entretien post-séquence, précise qu'elle en propose l'étude aux élèves de CM2 plusieurs fois dans l'année. Comme le montre le tableau ci-dessous, elle fait varier l'attention portée à chacune de ses composantes ( portrait physique, portrait psychologique) et ce en lien avec le propos de l'œuvre littéraire étudiée et le débat interprétatif envisagé.

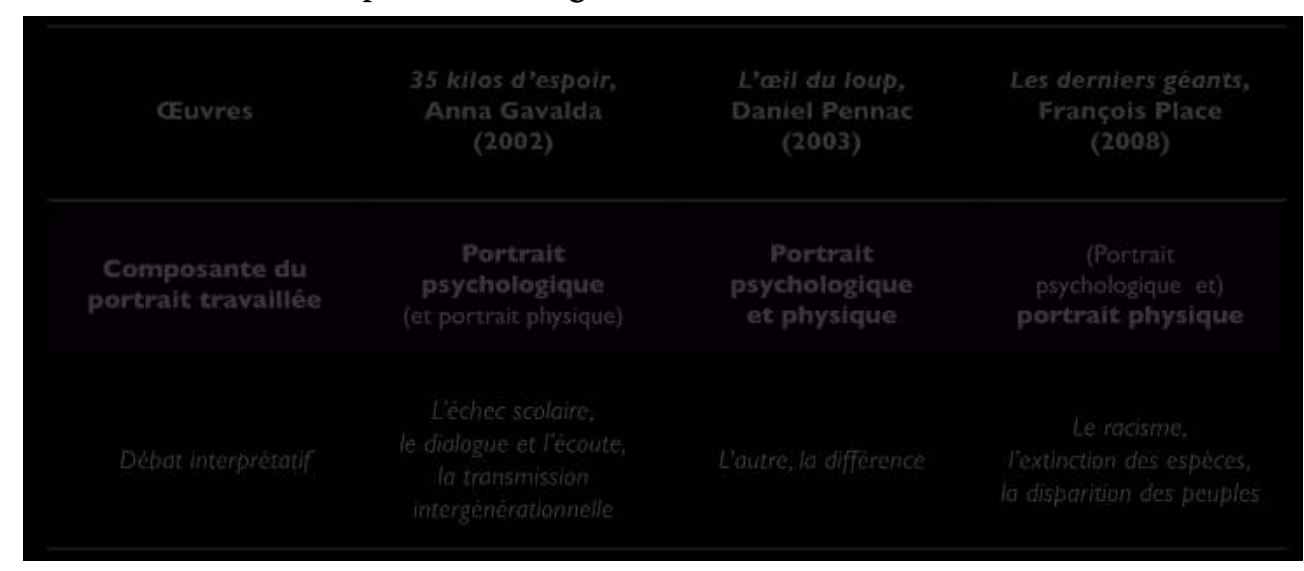

Effectivement, dans la progression telle qu'elle est ici pensée ${ }^{14}$, il est possible, pour chaque étude littéraire, de déterminer un niveau de formulation pour la pratique du portrait qui corresponde à une fonction différente de celui-ci dans la narration: explicative, régulatrice-transformationnelle, informative. On peut alors mobiliser autour du nom, notion-noyau pour la construction du portrait ou de la description, des savoirs et savoir-faire à mettre en œuvre dans l'écriture. On obtient alors la progression suivante, qu'il s'agit bien sûr d'exploiter en fonction de ce que les élèves savent ou ne savent pas (faire). 


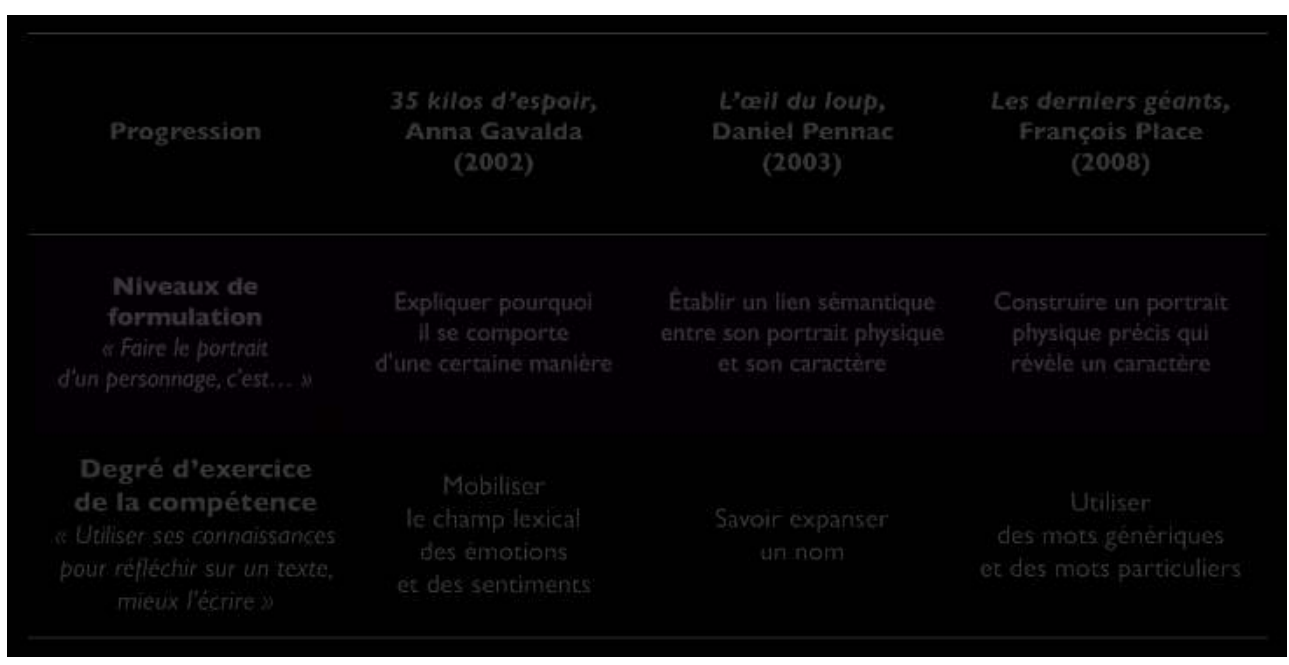

\section{Problématisation du modèle, modèle pour la problématisation}

21 En se présentant sous la forme d'un schéma organisé autour d'un vecteur temps linéaire, le modèle pour l'écriture en milieu scolaire proposé par Claudine Garcia-Debanc fait oublier que le temps didactique est une suite discontinue d'instants épars dans la séance, la séquence, l'année et que les élèves doivent pouvoir les relier les uns aux autres pour donner un sens aux activités scolaires qui leur sont proposées. Ce qui peut leur permettre de trouver de la cohérence dans cette multitude de moments, c'est non seulement de fédérer toutes les activités de la classe de français autour de l'écriture mais que cette écriture soit commandée par un problème dont ils ont à rechercher la solution, ce qui revient à problématiser l'écriture scolaire. Mais que s'agit-il au juste de problématiser ?

Bien évidemment, l'objet à écrire (interview, récit d'aventures). La problématisation concerne alors la dimension textuelle des productions; l'écriture s'envisage comme un objet d'apprentissage, le travail s'intéresse plutôt aux invariants des genres et discours pratiqués, il est ponctué par des moments forts d'institutionnalisation. La dynamique de l'écriture, par le jeu des critères textuels ou de réalisation, se déploie en un enchaînement problématique correspondant à divers niveaux de formulation; on y travaille des notions-noyaux; on y exerce des compétences à un certain niveau. Tous ces aspects sont décrits dans le modèle étudié ; ils apparaissent dans les diverses opérations déployées (" utiliser des critères ", " repérer des fonctionnements ", "élaborer, utiliser des outils ", " activités décrochées») et dans le caractère itératif du schéma, qui va de l'écriture aux multiples réécritures possibles ${ }^{15}$.

Mais il s'agit également de problématiser l'écriture scolaire elle-même et, pour ce faire, de l'utiliser non seulement comme un moyen d'évaluation formative ${ }^{16}$ ou sommative des acquis par l'enseignant, mais comme un véritable moyen de communication. C'est en donnant à l'écriture scolaire une dimension pragmatique que celle-ci peut, au fil des réécritures, par des négociations orales et écrites autour des écrits (des caricatures, par exemple), permettre aux élèves d'accompagner et de contrôler leurs productions, de les co-construire (Bucheton, 2014). En communiquant aussi avec l'École et les familles, l'écriture scolaire se donne un contexte familier qui permet de se tourner vers des objets réellement "étonnants», c'est-à-dire dignes d'intérêt parce que dotés d'enjeux 
importants (des enjeux cognitifs, culturels, psychiques, etc.). Quand elle joue ce rôle de communication entre tous les acteurs impliqués dans l'enseignement et l'apprentissage, l'écriture dépend plutôt des spécificités de la situation posée par le problème et de ses obstacles. Ceux-ci peuvent être autant d'occasions de collaborer, autrement que prévu, à la construction de ce qui est à écrire. Toutes choses que le Schéma systémique de la production écrite, en pratiquant l'ellipse des contextes autres que celui de «la classe » et celle de la place et du statut de l'oral, ne dit pas explicitement et qui appellent le recours à d'autres modèles théoriques, par exemple ceux de la problématisation.

Car c'est bien de la mise en tension de ces deux pôles (textuel/pragmatique) que l'écriture scolaire reçoit son sens, en assumant (au moins) deux fonctions hétérogènes : une fonction scolaire visant à faire rationnellement construire des savoirs et savoir-faire, à évaluer des apprentissages, à étalonner les élèves; une fonction sociale définie au regard du genre pratiqué (informer, divertir, enseigner...), mobilisant fortement les ressources de l'imaginaire. On sait que c'est plutôt du côté des pédagogies actives, comme la pédagogie Freinet, que les conditions ${ }^{17}$ semblent réunies pour que la problématisation de l'écriture puisse avoir lieu. Mais il s'agit là d' «expérience[s] atypique[s] dans le paysage scolaire français »(Reuter, 2007, p. 9). Dans les pratiques traditionnelles, la fonction de communication de l'écriture reste trop souvent absente ou sous le contrôle de l'enseignant, qui privilégie le travail sur la dimension textuelle des écrits. Ceux-ci deviennent alors des exercices scolaires d'application. On sait également que tous les genres ne se prêtent pas aussi aisément à une finalisation des productions écrites: par exemple, la pratique du récit d'aventures, qui ne fait intervenir qu'un destinataire fictif (le lecteur), s'avère de ce point de vue plus difficile ${ }^{18}$. Quelles que soient les raisons qui peuvent expliquer la résistance à un usage pragmatique de l'écriture - et sans doute sont-elles nombreuses - de fait, elles impliquent un fonctionnement de la classe qui ne donne pas aux élèves les conditions d'une réelle problématisation des savoirs et des pratiques langagières. Dans ce cas, le schéma systémique de la production écrite peut jouer une fonction proprement idéologique, en faisant croire à un processus auto-producteur de l'écriture et du scripteur là où n'existe qu'une distribution chronologique de tâches ponctuelles. 


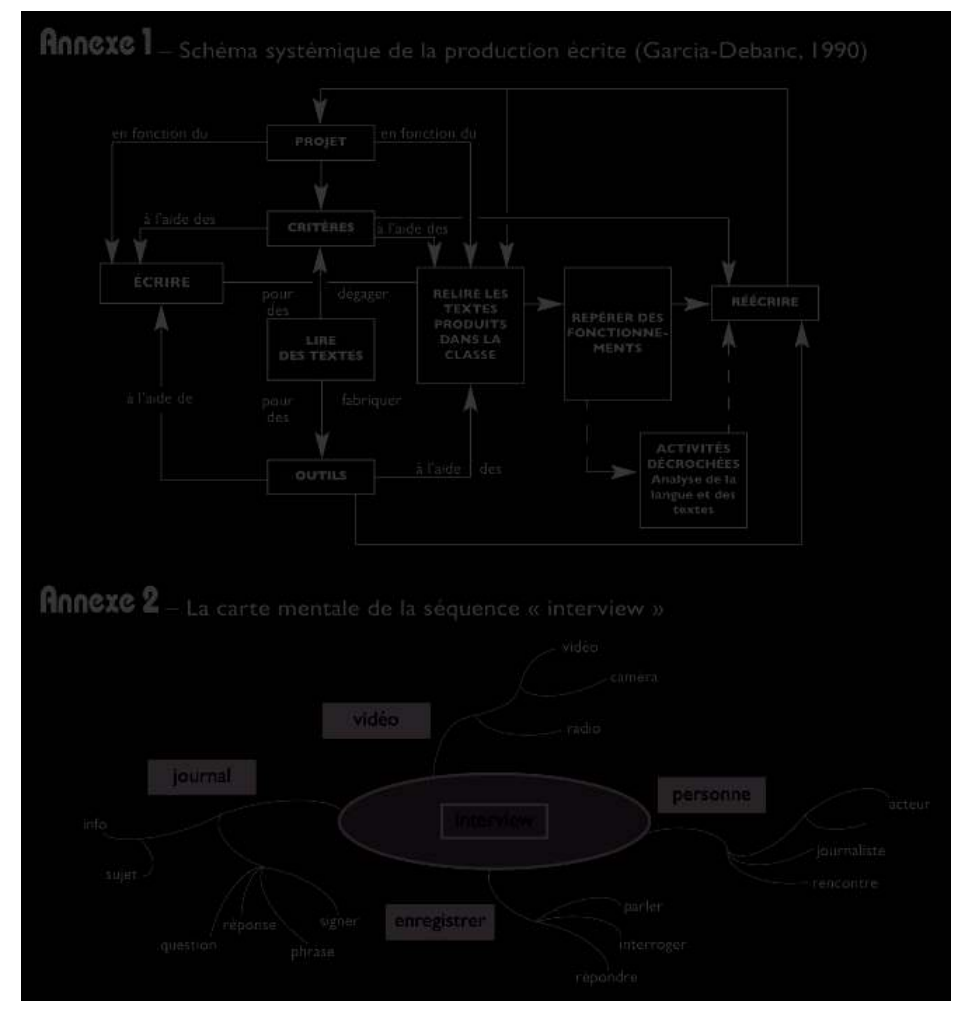

\section{BIBLIOGRAPHIE}

Bachelard, G. (1931). L'intuition de l'instant (Éd. 2014). Paris : Le Livre de Poche.

Bachelard, G. (1950). La dialectique de la durée (Éd. 2013). Paris : P.U.F.

Bucheton, D. (2014). Refonder l'enseignement de l'écriture. Vers des gestes professionnels plus ajustés du primaire au lycée. Paris : Retz.

Chabanne, J.-C. et Bucheton, D. (2002). Écrire en ZEP, un autre regard sur les écrits des élèves. Paris : Delagrave/C.R.D.P. de l'académie de Versailles.

Chalak, H. (2014). Difficultés de construction de savoirs et de textes problématisés en sciences de la Terre et pratiques enseignantes : étude d'une séquence ordinaire portant sur le magmatisme. Éducation et didactique, 8, 55-80.

Fabre, M. et Musquer, A. (2009). Comment aider les élèves à problématiser ? Les inducteurs de problématisation. Les sciences de l'Éducation - Pour l'ère nouvelle, 42(3), 111-129.

Fabre, M. (2014). De l'étonnement au problème. Éducation permanente, 200, 97-106.

Galluzzo-Dafflon, R. (2006). La description littéraire : objets et processus didactiques. Thèse de doctorat, Université de Rouen, $717 \mathrm{p}$.

Galluzzo-Dafflon, R. (2011). Projet d'écriture théâtrale et mises en œuvre de l'écriture d'invention au lycée. Recherches en didactiques, 12, 85-101. 
Galluzzo-Dafflon, R. (2015). Faire écrire un récit étiologique au CM2. Du questionnement épistémologique à l'enchaînement problématique. Éducation et didactique, 9(2), 9-26.

Garcia-Debanc, C. (1990). L'élève et la production d'écrits. Metz : C.A.S.U.M.

Halté, J.-F. (1992). La didactique du français. Paris : P.U.F.

Mas, M. (1987). Hors des critères, point de salut. Repères, 73, 1-8.

Nonnon, É. (2010). La notion de progression au cœur des tensions de l'activité d'enseignement. Repères, 41, 5-34.

Orange, C. (2007). Quel Milieu pour l'apprentissage par problématisation en sciences de la vie et de la terre ? Éducation et didactique, 1(2), 37-56.

Reuter, Y. (1995). Les relations et les interactions lecture-écriture dans le champ didactique, Pratiques, 86, 5-23.

Reuter, Y. (1998). La description en questions. In Y. Reuter (Dir.), La description. Théories, recherches, formation, enseignement. Villeneuve d'Ascq (pp. 33-59) : Septentrion.

Reuter, Y. (2002-2003). Quelques questions à propos des formalisations de l'écriture en didactique $\mathrm{du}$ français. Repères, 26-27, 21-30.

Reuter, Y. (2007). Comprendre les principes de fonctionnement de l'école « Freinet ». In Y. Reuter (Dir.), Une école Freinet (pp. 15-30). Paris : L’Harmattan.

\section{NOTES}

1. Il s'agit bien d'une description dans le sens où le schéma «fait voir » (Reuter, 1998, p. 37) ce processus, autrement dit «construit des informations qui donnent l'impression de visualiser ce dont il est question» (p. 38), ce qui correspond à « la visée centrale» (p. 34) de la description.

2. Dans cet article, Reuter interroge principalement la nécessité d'avoir une modélisation de l'écriture en didactique du français et les conceptions sur lesquelles celle-ci peut reposer.

3. La problématisation du temps didactique est dans ce cas opérée à partir $d u$ modèle chronologique présenté dans ce numéro par Michel Fabre.

4. Les types d'activités reconnus pendant l'observation de la séquence en centre-ville sont l'écriture, la mise en commun, la recherche, les échanges récurrents et progrédiants, l'élaboration de critères, les activités organisationnelles; en milieu rural, le temps didactique observé est réparti entre l'écriture, la relecture critique des premiers jets, le compte rendu de l'évaluation magistrale, l'élaboration de critères, la lecture de la grille de relecture.

5. Pour explorer les conditions de cette hypothétique cohésion, je me référerai donc plutôt au deuxième modèle de la problématisation, le modèle expressif (voir l'article de Michel Fabre dans ce numéro).

6. Désormais TAP.

7. L'écriture peut être définie comme « une pratique sociale, historiquement constituée, mettant en jeu des savoirs, des représentations, des investissements, des valeurs ainsi que des opérations physiques, psychologiques et cognitives complexes, visant à construire du sens dans la production d'un écrit » (Reuter, 1995, p. 7).

8. On peut rappeler que l'interview est l'entretien d'un journaliste avec une personne (généralement en vue) qui accepte de répondre, pour les besoins d'un article ou d'une émission radiophonique ou télévisée, à des questions d'ordre professionnel ou personnel. Cette personne se doit donc d'être « intéressante » d'un certain point de vue. 
9. Dans l'entretien post-séquence, le professeur souligne l'importance quantitative des familles monoparentales et recomposées. Le blog pourrait, selon lui, aider la communication entre l'École et la famille, rendue plus délicate dans les deux cas.

10. Cette carte mentale est élaborée en deux temps : - le recueil individuel de noms librement associés par l'élève à celui d' "interview »; - la répartition de ces noms sous des mots-thèmes pouvant les subsumer.

11. En didactique du français, l'utilisation de caricatures est également à l'étude dans le cadre de la lecture littéraire. Voir Huchet, C. \& Simon, F. (2014). Texte sur les caricatures (document de travail pour le séminaire « Inducteurs », CREN, 28 novembre).

12. Il y a "enchaînement problématique » quand une ou plusieurs des conditions trouvées pour solutionner un problème deviennent les données d'un second, dans un nouveau questionnement. Pour un exemple en didactique du Français, voir Galluzzo-Dafflon, 2015.

13. Dans les faits, ce critère (induit par le travail régulier, dans la séquence, sur le schéma narratif et le résumé) a surtout agi sur le découpage - le plus souvent motivé - du récit en cinq paragraphes.

14. Cette progression est tributaire des œuvres choisies pour objets d'étude. Il est bien entendu possible d'en déterminer d'autres, plus centrées sur les critères de genre et leur évolution historique (Galluzzo-Dafflon, 2006).

15. Pour un exemple de travail dans ce sens à propos du récit étiologique, voir Galluzzo-Dafflon, 2015.

16. Je rappelle que c'est la vocation première du schéma de Mas.

17. On peut considérer que ces conditions correspondent aux six principes relevés par Reuter (2007, p. 16-19): l'école est centrée sur les apprentissages; l'école fait l'objet d'un travail d'institutionnalisation; l'école est considérée comme une société; cette société s'appuie sur un idéal démocratique; cette société se construit une culture commune; cette société ne vit pas en autarcie.

18. C'est la raison pour laquelle les enseignants recourent en général à des projets d'écriture visant la publication des productions sous la forme de livres ou fascicules, de spectacles. Pour un exemple dans le secondaire, voir Galluzzo-Dafflon (2011).

\section{RÉSUMÉS}

Après avoir rappelé le contexte d'émergence du Schéma systémique de la production écrite (Garcia-Debanc, 1990) et quelques critiques dont il a été l'objet, l'article s'intéresse au temps didactique et à sa représentation graphique. Deux séries d'observation en CM2 montrent que ce temps, hautement problématique, nécessite une mise en cohérence des instants constituant la durée d'une période de travail, par une attention à la dimension textuelle et pragmatique des productions. En occultant la seconde de ces dimensions, le Schéma systémique est donc susceptible de jouer une fonction idéologique.

After mentioning the context in which the Schéma systémique de la production écrite (GarciaDebanc, 1990) stood out, and some of the appreciations it was the subject of, the article focuses on the didactic time and on its graphical representation. Two series of observations of last-yearpupils in primary school demonstrate that this highly problematical time calls for the coherence of the moments which a working period is made up of, for as long as this period may last, by taking into account the textual and pragmatic aspects of the pupils' works. The Schéma 
systémique loses sight of the latter aspect, which is why it is likely that it would fulfil an ideological function.

INDEX

Mots-clés : didactique du Français, écriture, problématisation, temps didactique

Keywords : didactic time, didactics of French, problem-posing, writing

\section{AUTEUR}

\section{ROSINE GALLUZZO-DAFFLON}

Maître de Conférences, ESPE de l'Académie de Nantes, site d'Angers, CREN, EA 2661 